\title{
Probability of the resistive state formation caused by absorption of a single-photon in current-carrying superconducting nano-strips
}

\author{
Alexei Semenov, Andreas Engel $*$ and Heinz-Wilhelm Hübers \\ DLR Institute of Planetary Research, Rutherfordstr. 2, 12489 Berlin, Germany \\ Konstantin Il'in and Michael Siegel \\ Institute of Micro- and Nano-Electronic Systems, \\ University of Karlsruhe, Hertzstr. 16, 76187 Karlsruhe, Germany
}

\begin{abstract}
We have studied supercurrent-assisted formation of the resistive state in nano-structured $\mathrm{Nb}$ and $\mathrm{NbN}$ superconducting films after absorption of a single photon. In amorphous narrow NbN strips the probability of the resistive state formation has a pronounced spectral cut-off. The corresponding threshold photon energy decreases with the bias current. Analysis of the experimental data in the framework of the generalized hot-spot model suggests that the quantum yield for near-infrared photons increases faster than the photon energy. Relaxation of the resistive state depends on the photon energy making the phenomenon feasible for the development of energy resolving singlephoton detectors.

PACS numbers: $74.78 .-\mathrm{w}, 42.50 . \mathrm{Nn}$
\end{abstract}

\section{INTRODUCTION}

A recently proposed hot-spot scenario of the resistive state formation in a thin, narrow, current-carrying superconducting strip due to absorption of a single infrared photon ${ }^{1}$ was subsequently suggested as a mechanism of single-photon detection ${ }^{2.3}$ in $\mathrm{NbN}$ microbridges. Although soon after realization such detectors found applications, the first experimental evidence of the envisaged mechanism has been reported only a year ago $\underline{\underline{4}}$. The hotspot mode $\mathrm{l}^{1}$ describes the formation and the following evolution of a normal hot-spot around the absorption site in an infinite homogeneous superconducting film where a single photon has been absorbed. The energy of the captured photon is quickly shared among a large number of electrons via electron-electron and electron-phonon interaction leading to an avalanche-like multiplication ${ }^{5}$ of non-equilibrium quasiparticles (QP). The normal spot appears when the excess QP concentration becomes large enough in order to locally destroy superconductivity. At some stage the normal spot reaches the maximum size that is a trade-off between quasiparticle multiplication and their diffusion out of the absorption site. In a narrow strip carrying a small sub-critical current, no resistive state is formed unless the maximum size of the normal spot $A_{n}$ exceeds the strip width. If the spot-size is less than the strip-width, the resistive state may appear due to a supplementary action of the bias current that should have a density $j$ close to the critical current density $j_{C}$. Once the normal spot becomes larger than the coherence length, the supercurrent is expelled from the spot. The effective cross-section available for the current flow decreases and the actual current density may exceed the critical current density. When this happens, a normal domain spans the entire cross-section of the strip that causes a voltage pulse developing between the strip ends. The number of photons, which result in a voltage response, related to the number of photons crossing the geometrical area of the strip is a measure of the quantum efficiency (probability) of the resistive state formation. If $A_{n}$ is not sufficiently large in order to enhance the current density to its critical value, there is still a finite probability of the resistive state formation due to multi-photon or fluctuation assisted events ${ }^{2.3}$. Since $A_{n}$ increases with the photon energy there should be a current dependent photon energy $\epsilon_{0}$ that demarcates these two regimes of the resistive state formation. The threshold energy $\epsilon_{0}$ is defined by the simple geometric criteria $A_{n}\left(\epsilon_{0}\right) / w=1-\left(j / j_{C}\right)$ where $w$ is the width of the strip. For photon energies $\epsilon>\epsilon_{0}$ but smaller than $h \nu_{0}$ ( $\nu_{0}$ is the plasma frequency), the quantum efficiency should equal the probability of photon absorption and should not, therefore, depend on the photon energy. At $\epsilon<\epsilon_{0}$ the quantum efficiency is dominated by multiphoton and fluctuation assisted events, which generally have a smaller probability.

So far the cut-off of the quantum efficiency at $\epsilon \approx$ $\epsilon_{0}$ has not been experimentally observed, instead a monotonous decrease of the quantum efficiency with the photon wavelength has been reported ${ }^{3}$. Knee-like features have been found in the overall exponential dependence of the quantum efficiency on the bias current. Although they were attributed to the above discussed cutoff, the concluded size of the normal spot was far too large in comparison to model estimates ${ }^{6}$. The photon absorption in a superconductor obeys Poisson statistics, in that the probability of absorbing simultaneously $n$ photons from a weak photon flux is proportional to the flux intensity to the power of $n$. Variation of the quantum efficiency corresponding to $n=1,2$ and 3 have indeed been found ${ }^{2}$ in $\mathrm{NbN}$ microbridges. However, this observation would generally manifest quantum nature of any detector and is not uniquely specific to any particular response mechanism. A time delay of the resistive state formation 
in $\mathrm{NbN}$ meander lines has been recently reported ${ }^{4}$. The delay was attributed to the supplementary action of the bias current in the formation of the normal domain but the quantitative description implied the initial normal spot much larger than the hot-spot model predicts.

In this work we report measurements of the quantum efficiency of the resistive state formation in narrow amorphous strips made from $\mathrm{Nb}$ and $\mathrm{NbN}$ films. A reduced electron diffusivity and a reduced density of electronic states in $\mathrm{NbN}$ allowed us to observe the predicted cut-off of the quantum efficiency and to evaluate the intrinsic efficiency of the electron multiplication. We refine the hot-spot model and show that the resistive state formation in the absence of fluctuations may be triggered by a single photon even though no normal spot appears. This approach eliminates contradictions within the earlier published experimental data and provides a quantitative explanation of how fluctuations enhance the quantum efficiency beyond the cut-off. We also present recent results showing that the resistive state created by a photon "remembers" its energy, i.e. the effect has energy resolving capability.

\section{EXPERIMENT}

\section{A. Sample preparation and measurement technique}

Experimental results were obtained with narrow meander lines made from either $\mathrm{Nb}$ or $\mathrm{NbN}$ thin films. Meanders covering an area of $4 \times 4 \mu \mathrm{m}^{2}$ were used in order to increase optical coupling with near infrared photons. Contrary to the conventional technique, our modified $\mathrm{NbN}$ films were prepared by dc magnetron sputtering of an $\mathrm{Nb}$ target in an $\mathrm{Ar}+\mathrm{N}_{2}$ gas admixture that had a reduced partial pressure of $\mathrm{N}_{2}$. The deposition regime in combination with relatively high sputtering rates of about $1.2 \mathrm{~nm} / \mathrm{sec}$ resulted in amorphous films with a nitrogen content smaller than in the stoichiometric composition. Films with a nominal thickness of $5 \mathrm{~nm}$ were deposited on sapphire substrates kept at room temperature. Mostly because of the nitrogen deficiency, the superconducting transition in $5 \mathrm{~nm}$ thick films occurred at $\approx 6$ $\mathrm{K}$ that is about one third of the transition temperature of bulk NbN. Moreover, the transition temperature was partly suppressed due to the proximity effect ${ }^{7}$ between the central superconducting layer of the film and both the non-superconducting oxidized surface on one side and the film-substrate boundary on the other side. Films were patterned using electron-beam lithography and ion milling. Our $\mathrm{Nb}$ films had a thickness of $10 \mathrm{~nm}$ and were prepared on Si substrates by dc magnetron sputtering in pure Ar atmosphere. Films were patterned using electron-beam lithography and reactive ion etching. The line width was $84 \mathrm{~nm}$ and $98 \mathrm{~nm}$ and the normal state resistivity just above the superconducting transition was $6 \cdot 10^{-6} \Omega \mathrm{cm}$ and $6.7 \cdot 10^{-4} \Omega \mathrm{cm}$ for $\mathrm{Nb}$ and $\mathrm{NbN}$ meanders, respectively. Due to a partial damage of strip edges

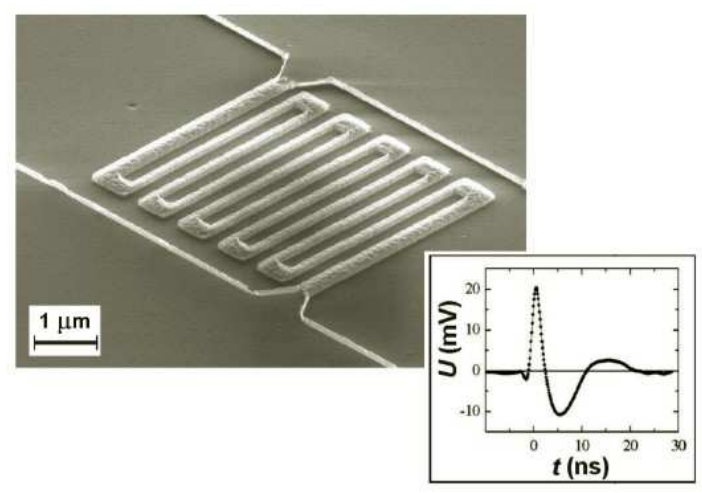

FIG. 1: SEM photo of a representative $\mathrm{Nb}$ meander on $\mathrm{Si}$ substrate. Visible fringes at the edges of the structure are formed by photoresist left for device protection. Inset shows a typical voltage pulse as recorded by the oscilloscope.

by argon ions during the etching process, a portion of the meander line near the edges became normal. These normal areas further decreased ${ }^{8}$ the transition temperature. For both materials the transition temperature $T_{C}$ of the meander varied between 4 and $5 \mathrm{~K}$. An image of a representative $\mathrm{Nb}$ meander made with a scanning electron microscope is shown in Fig. [1

In order to ensure a sufficiently high quality of our $\mathrm{NbN}$ structures we evaluated their superconducting parameters relevant to the formation of a critical current state. Measuring the temperature variations of the second critical magnetic field $B_{c 2}$ near $T_{C}$, we concluded the diffusion coefficient $D=0.35 \mathrm{~cm}^{2} \mathrm{sec}^{-1}$. This relatively low electron diffusivity assured that the meanders were in the diffusive, dirty limit. Extrapolating the linear temperature dependence of $B_{c 2}$ near $T_{C}$, we found $B_{c 2}(0)=9.4 \mathrm{~T}$ and the zero-temperature coherence length $\xi_{0}=\left(\Phi_{0} / \pi B_{c 2}(0)\right)^{1 / 2} \approx 8.5 \mathrm{~nm}$. The density of electronic states $N_{0}=2.2 \cdot 10^{24} \mathrm{~m}^{-3} \mathrm{~K}^{-1}$ is then deduced from the Einstein's relation $N_{0}=1 /\left(e^{2} \rho D\right)$ where $\rho$ stands for the normal-state resistivity. Further assuming a classical energy gap $\Delta_{0}=1.76 k_{B} T_{C}$, we estimated the London penetration depth $\lambda_{L}(0)=1200$ $\mathrm{nm}$. Since the density of states was an important parameter for the evaluation of the size of the normal spot, we cross-checked it measuring the critical current density as a function of temperature. Because the width of our structure was well below the London penetration depth, the supercurrent density did not vary across the meander line. To derive the critical current density from the measured values of the critical current we used the electrical cross-section of the meander. Due to the proximity effect at the lateral interfaces and the damaged edges the electrical cross-section was almost one half ${ }^{8}$ of the geometrical cross-section. The best fit of the measured temperature dependence with $j(T)=$ $3.27 e N_{0} \Delta_{0}\left(k_{B} T_{C} D / h\right)^{1 / 2}\left(1-\left(T / T_{C}\right)^{2}\right)\left(1-\left(T / T_{C}\right)^{4}\right)^{1 / 2}$ resulted in $N_{0}=2.7 \cdot 10^{24} \mathrm{~m}^{-3} \mathrm{~K}^{-1}$ in agreement with the density of states inferred from the film resistivity. Practi- 
cal coincidence of the measured critical current with the depairing critical current evidences amorphous structure with a large concentration of defects that play a role of pinning centers. The granularity, if any, does not influence the critical current and should not therefore effect diffusion of electrons.

The substrate carrying the meander was thermally anchored to the cold plate of a $\mathrm{He}^{4}$-bath cryostat and was operated at temperatures ranging from about $1.8 \mathrm{~K}$ to approximately $3.0 \mathrm{~K}$. The meander was illuminated using an incandescent light source. To enable spectral measurements, the light was passed through a prism monochromator. The meander was voltage-biased through a voltage divider mounted inside the cryostat. Estimated time constant of the bias was $\tau_{B}=0.5 \mathrm{~ns}$. The response to illumination was in the form of a random sequence of voltage pulses each of them signaling either absorption of a photon or a dark count event. Voltage pulses were amplified using broadband microwave amplifiers (noise temperature $6 \mathrm{~K}$, band-pass from $0.1 \mathrm{GHz}$ to $1.6 \mathrm{GHz}$ ) and then guided either to a $250 \mathrm{MHz}$ bandwidth/1 GHz sampling rate oscilloscope or to a $200 \mathrm{MHz}$ bandwidth voltage-level counter. Inset in Fig. [1 shows the resulting signal transient recorded with the oscilloscope. The mean photon count rate, that is proportional to the quantum efficiency, was normalized to the light intensity measured with either a silicon photodiode or a PbSe photodiode (at longer wavelengths). For dark count measurements the optical entrance to the cryostat was blocked completely.

Relaxation of the resistive state, and consequently the voltage pulse across the meander, should last longer when initiated by a photon with a larger energy. Material parameters of $\mathrm{NbN}$, in particular electron-phonon interaction time, suggest that at $2 \mathrm{~K}$ the duration of the voltage pulse should be in the sub-nanosecond range. Bearing in mind the band-pass of our read-out electronic, we expect it to integrate pulses in real time. The amplitude of the signal transient after the amplifier chain should then correlate with the energy of the absorbed photon. Varying the discriminator level of the counter, we measured the statistical distribution of the signal amplitude and retrieved the mean value and the dispersion.

\section{B. Experimental data}

The normalized quantum efficiencies of the resistive state formation for $\mathrm{Nb}$ and $\mathrm{NbN}$ meanders are compared in Fig. 2] For both materials it was measured at $1.8 \mathrm{~K}$ using a current $I=0.9 I_{C}$, where $I_{C}$ is the critical current at the operation temperature. The quantum efficiency for the $\mathrm{Nb}$ meander drops continuously with the increase of the wavelength showing no threshold features while the $\mathrm{NbN}$ meander demonstrates an almost constant efficiency of $\approx 1 \%$ up to wavelengths that exceed $2 \mu \mathrm{m}$. Obviously, the threshold photon energy for $\mathrm{Nb}$ falls out of our experimental range and should correspond to a wavelength less than $0.25 \mu \mathrm{m}$. According to the model described

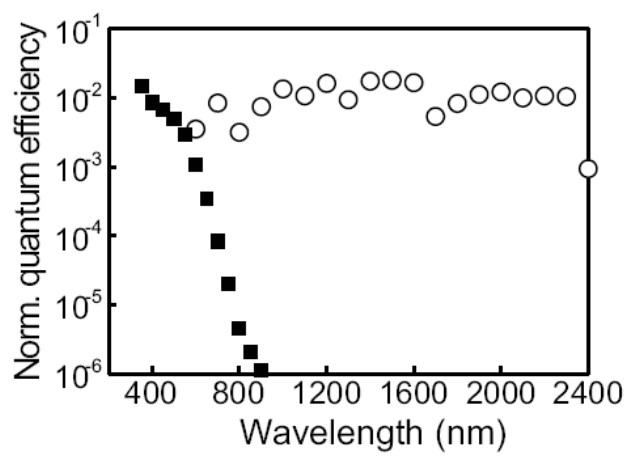

FIG. 2: Normalized quantum efficiency for Nb (closed symbols) and $\mathrm{NbN}$ (open symbols) meanders.

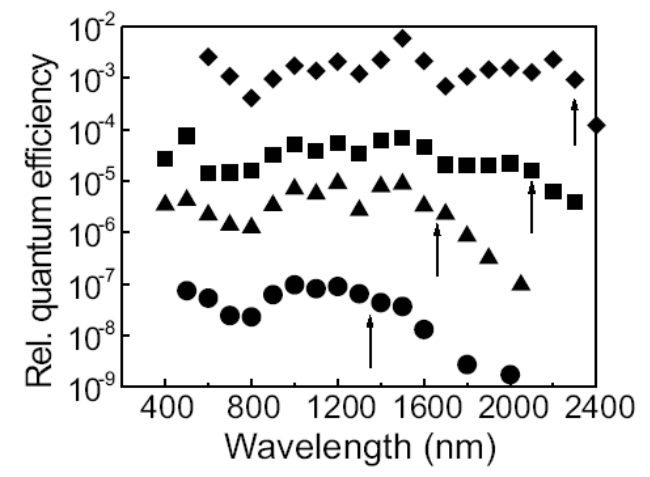

FIG. 3: Relative quantum efficiency for a representative $\mathrm{NbN}$ meander biased with currents $0.6 I_{C}, 0.77 I_{C}, 0.8 I_{C}$ and $0.89 I_{C}$. (from bottom to top). Arrows mark wavelengths corresponding to the cut-off at each bias current. For convenience, the data sets are shifted arbitrarily along the vertical axis.

in the next section, the threshold wavelength should be proportional to $\rho(D)^{1 / 2}\left(\Delta_{0}^{2} d\right)^{-1}$. Given the difference in the resistivity, the film thickness and the electron diffusivity, more than an order of magnitude difference in the threshold photon energy between $\mathrm{Nb}$ and $\mathrm{NbN}$ meanders is easily understood. In the sections that follow we concentrate exclusively on $\mathrm{NbN}$ meanders.

A decrease of the current brings to light the cut-off of the quantum efficiency in the NbN meanders. This can be seen in Fig. 3 that shows the relative quantum efficiency of a representative $\mathrm{NbN}$ meander measured at $2 \mathrm{~K}$ with different bias currents. For each current there is a plateau in the wavelength dependence of the quantum efficiency that is followed by a drop as the wavelength increases. The wavelength corresponding to the cut-off increases with the bias current. Alternatively, varying the current through a meander exposed to illumination at a fixed wavelength, one should also be able to observe the cut-off of the photon count rate. However, this way additional uncertainties are introduced in detecting the 


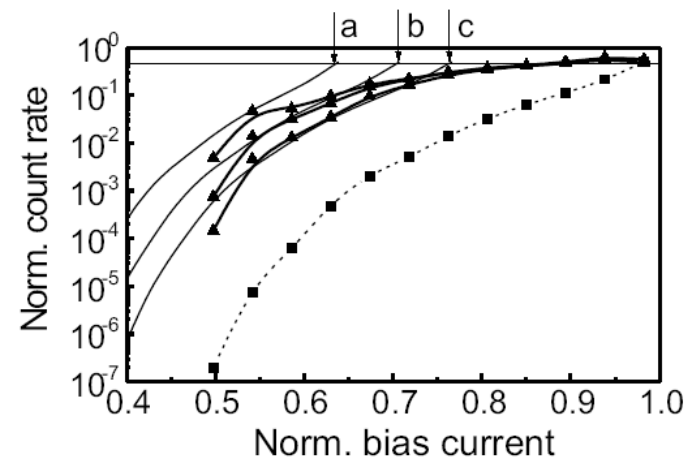

FIG. 4: Normalized photon count rates for wavelengths $0.8 \mu \mathrm{m}, 1.4 \mu \mathrm{m}$ and $2 \mu \mathrm{m}$ (thick solid lines, from top to bottom) and dark count rate (dashed line) both measured for the $\mathrm{NbN}$ meander at $\approx 0.5 T_{C}$. The largest absolute count rate was $5 \cdot 10^{7} \mathrm{sec}^{-1}$. Arrows mark the currents corresponding to the cut-off at: $\mathrm{a}-0.8 \mu \mathrm{m} ; \mathrm{b}-1.4 \mu \mathrm{m} ; \mathrm{c}-2 \mu \mathrm{m}$. Thin solid lines approximate the photon count rates beyond the cut-off.

threshold energy. The current destroys the symmetry of the energy states of superconducting electron pairs. The pair states enter the energy gap and the minimal excitation energy for some electron pairs becomes smaller than the zero-current energy gap. The excitation energy decreases with the bias current and becomes zero when the current density reaches the depairing critical current density. The decrease of the minimum excitation energy with the bias current smears the threshold feature and introduces a current dependence of the photon count rate above the cut-off. Fig. 4 shows the current dependence of the photon count rate (thick solid lines) for different photon energies. Indeed, when the bias current is close to the critical value, all curves merge and the count rate slowly varies with the current. The curve corresponding to a particular wavelength starts to deviate from this common dependence at a current that increases with the wavelength. We associate the deviation from the common dependence with the cut-off of the quantum efficiency. Threshold values of the current are marked by arrows. The current dependence of the photon count rate beyond the cut-off was approximated (thin solid curves) using the current dependence of the dark count rate shown as the dotted line in Fig. 4 The connection between the photon counts and dark counts will be discussed below.

\section{MODEL REFINEMENT}

Applying to our experimental data (Fig. 3) the geometrical criteria of the hot-spot model, we find the maximum normal-spot size of $8.5 \mathrm{~nm}$ created by a photon with the $1.2 \mu \mathrm{m}$ wavelength. An analytical approximation ${ }^{6}$ of the numerical solution of the two dimensional diffusion problem gives a close value of $\approx 9 \mathrm{~nm}$. According to

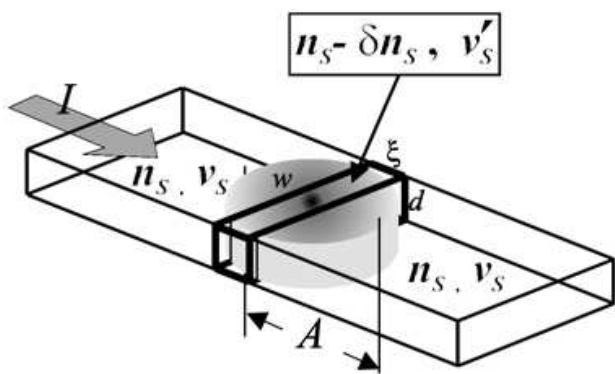

FIG. 5: Schematics of the superconducting strip-line carrying a current $I$. Bold lines demarcate the smallest volume where a reduction of the superconducting electron pairs causes a change of their mean velocity. Grey cylinder depicts the cloud of nonequilibrium quasiparticles with the size $A$.

this approximation, even smaller normal spot should appear in conventional $\mathrm{NbN}$ films ${ }^{2.3}$ because they have a larger density of states, a larger energy gap and a larger electron diffusivity. However, all those spots are comparable or less than the coherence length suggesting that no resistive state should appear in response to photons with larger wavelength. Contrary, our experimental data show that not only the resistive state appear but there is a well pronounced spectral cut-off corresponding to wavelengths larger than $1.2 \mu \mathrm{m}$. In order to eliminate this discrepancy, we will refine the model considering the effect of quasiparticles, which are not confined in the normal spot.

Assume a strip made of a superconducting film carries a supercurrent $I<I_{C}$ as it is shown in Fig. 5 The film thickness $d$ is chosen smaller than both the line width $w$ and the coherence length $\xi$ assuring the applicability of a two-dimensional diffusion model for nonequilibrium electrons. The small film thickness results in a large magnetic penetration length that exceeds the strip width even at temperatures well below the superconducting transition temperature. In this geometry, the magnetic field of the supercurrent may be neglected and, in the absence of magnetic vortices, the local supercurrent density should be constant throughout the cross-section of the strip. However, since our strips are wider than the coherence length, one can not exclude the presence of vortices. If there are vortices they should obey a Kosterlitz-Thouless (KT) topological transition. Square resistance of our $\mathrm{NbN}$ films was always smaller than the resistance quantum $h / e^{2}$ resulting in the $\mathrm{KT}$ transition temperature ${ }^{9}$ only slightly below $T_{C}$. At typical operation temperatures $\approx T_{C} / 2$ all vortices are then bundled into pairs and do not contribute to the resistance unless the bias current is very close to the critical current. Although bundled vortices modulate the supercurrent density, we will neglect this current distribution for a while and associate the local supercurrent density $j=e n_{s} v_{s}$ with the mean velocity $v_{s}$ and the mean density $n_{s}$ of paired electrons. The local current state may noticeably change 
only if $n_{s}$ changes over a distance $\xi$ or larger along the current path, shorter perturbations are tunnelled by electron pairs without energy dissipation. The smallest volume of the strip relevant to a current change, the $\xi$-slab, is marked in Fig. [5 with bold lines. If $n_{s}$ in the $\xi$-slab decreases by an amount $\delta n_{s}$, the mean pair velocity increases and becomes

$$
v_{s}^{\prime}=\frac{n_{s}}{n_{s}-\delta n_{s}} v_{s}
$$

as required by charge flow conservation. The characteristic conversion time of the pair velocity practically equals the Ginzburg-Landau relaxation time $\approx h / \Delta$ ( $\Delta$ is the temperature dependent energy gap) and is small compared to the electron thermalization time $\tau_{t h}$. Thus, the mean velocity in the slab instantaneously follows changes of the pair density. The $\xi$-slab switches into the normal state if the pair velocity exceeds the critical value $v_{s c}$ that corresponds to the critical current density $j_{C}=e n_{s} v_{s c}$ in the absence of perturbations.

The concentration of nonequilibrium electrons $C(r, t)$ at a distance $r$ from the photon absorption site evolves in time due to multiplication of electrons and their diffusion

$$
C(r, t)=\frac{M(t)}{4 \pi D d t} \exp \left(-\frac{r^{2}}{4 D t}\right),
$$

where $M(t)$ is the time dependent number of nonequilibrium electrons. At $t=\tau_{t h}$ nonequilibrium electrons have thermalized to the energy level $\Delta$ effectively becoming quasiparticles. Their number reaches the maximum value $M\left(\tau_{t h}\right)=\zeta \epsilon / \Delta$ where $\zeta \leq 1$ is the efficiency of the QP multiplication. After the thermalization time $\tau_{t h}$ has elapsed and before subgap phonons appear, the concentration of nonequilibrium quasiparticles locally equals the reduction of superconducting electrons $\delta n_{s}$. Assuming that well below the transition temperature $n_{s} \approx N_{0} \Delta$, one can rewrite (1) and find the smallest number of nonequilibrium QP that is sufficient for switching the $\xi$-slab into the normal state

$$
\delta N^{*}=N_{0} \Delta \xi w d\left(1-I / I_{C}\right) .
$$

If the spread of the QP cloud is comparable to the coherence length, all quasiparticles contribute to the change of the condensate velocity and $\delta n_{s}=M\left(\tau_{t h}\right) /(w d \xi)$. If the cloud is larger (see Fig. 5), only quasiparticles confined within the $\xi$-slab have to be taken into account. The size $A(t)$ of the electron cloud is twice the radius of the spot that confines all nonequilibrium electrons. Equating $M(t)$ to the integral of the electron concentration (Eq. (2)) over the cylinder with the radius $A(t) / 2$ and the thickness $d$, we find the time dependent size of the electron cloud $A(t)=4[D t \ln (M(t))]^{1 / 2}$. If $M\left(\tau_{t h}\right) \geq 100$ and typical parameters of $\mathrm{NbN}$ films are considered, $A(t)$ exceeds the coherence length already at an early thermalization stage $t<\tau_{t h}$. For $A \gg \xi$ the absolute number $\delta N$ of nonequilibrium electrons confined to the $\xi$-slab can be

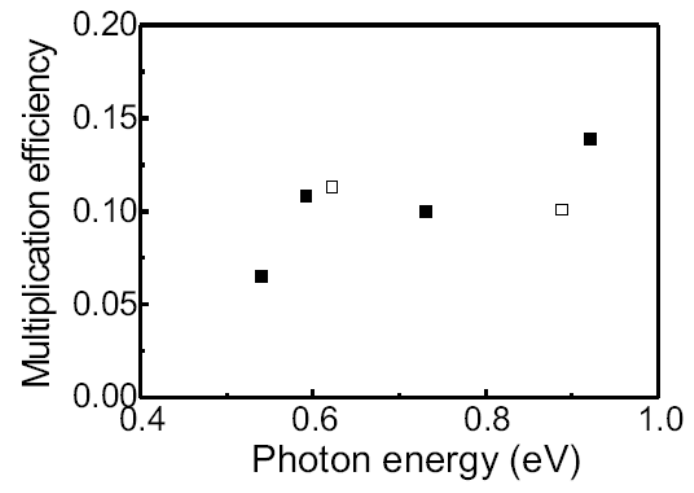

FIG. 6: Multiplication efficiency of quasiparticles for different photon energies concluded from current (open symbols) and spectral (closed symbols) dependence of the photon count rate.

evaluated analytically. Integrating the electron concentration (Eq. (2)) over the slab results in

$$
\delta N=M(t) \frac{\xi}{\sqrt{\pi D t}}
$$

The number of nonequilibrium electrons in the slab peaks at $t \approx \tau_{t h}$ when nonequilibrium electrons can already be treated as quasiparticles. The cut-off of the quantum efficiency occurs when the maximum number of nonequilibrium QP in the slab reaches $\delta N^{*}$. The criteria becomes $\delta N\left(\tau_{t h}\right)=\delta N^{*}$ that allows to determine the efficiency of the quasiparticle multiplication

$$
\zeta=\frac{N_{0} \Delta^{2} w d \sqrt{\pi D t}\left(1-I / I_{C}\right)}{\epsilon} .
$$

Using material parameters of our films, we estimated an efficiency of $\approx 0.1$ in the near infrared range.

The maximum size of the actual normal spot $A_{n}$ can be estimated solving the equation $C\left(A_{n} / 2, \tau_{t h}\right)=N_{0} \Delta$ and using the above value for the multiplication efficiency. In our films it turns out that $A_{n}<\xi$ and according to the hot-spot model the formation of a resistive state should not be triggered. When applied to the conventional films with a stoichiometric composition ${ }^{2.3}$ this analysis even suggests that no normal spot at all is formed. Finally, it is worth noting the meaning of the size of the normal spot that is defined by the criteria $A_{n} / w=\left(1-I / I_{C}\right)$ : it is the diameter of a cylinder that would confine all nonequilibrium quasiparticles at $t=\tau_{t h}$ if their concentration would have been uniform and equal $N_{0} \Delta$.

Assuming that the values of the bias currents and wavelengths indicated by arrows in Figs. 3 and 4 define the cut-off that satisfies the criteria $\delta N=\delta N^{*}$, we calculated for each pair of current and wavelength the efficiency of QP multiplication. Fig. [6] shows the efficiency $\zeta$ for different photon energies. The data points presented by open symbols were obtained from the current dependence of the photon count rate (Fig. (4)). They are less 
accurate (see the discussion above) than the data obtained from the spectral dependence of the photon count rate. Therefore, we omitted the data point derived from the count rate of $0.8 \mu \mathrm{m}$ photons. The efficiency of the QP multiplication increases with the photon energy. Although the physical reason for the increase is not quite clear, we speculate that it might be due to a splitting of the electron cascade into the electron and phonon branches. The splitting occurs 5 around the Debye energy, which is slightly less than photon energies used in our experiment.

\section{FLUCTUATIONS AND QUANTUM EFFICIENCY BEYOND THE CUT-OFF}

Besides the fundamental interest in mechanisms, which result in dark counts in two-dimensional films, fluctuations are important for sensor applications where small photon fluxes have to be detected. Depending on the cross-section dark counts in one-dimensional superconductors may originate from the spontaneous formation of either classical ${ }^{10}$ or quantum ${ }^{11}$ phase-slips. When the cross-section confining the current path extends over the coherence length, dark counts can appear due to discrete fluctuations of the superconducting order parameter 12.13 and/or number fluctuations in the gas of bundled magnetic vortices 14 . It has been observed in many experiments that the dark count rate strongly depends on the bias current. Since at temperatures well below $T_{C}$ the density of the superconducting condensate does not vary much with the bias current $\frac{15}{15}$, we suggest that the current dependence of the dark count rate is connected only to the velocity of the superconducting electrons. In particular, the velocity controls the minimal excitation energy of the electron pairs. Although variations of the pair density modify the current dependence of the dark count rate, experimental data show ${ }^{13}$ that this modification is a week factor in comparison to the current dependence itself. According to Eq. (1), the bias current corresponding to the spectral cut-off at a fixed photon energy can be presented as $I^{*}=I_{C}\left(1-\delta n_{s} / n_{s}\right)$. For currents $I<I^{*}$ a photon counting event can be thought of as a dark count event associated with that portion of the superconducting strip, where the density of electron pairs has been decreased due to photon absorption. The elevated pair velocity in this portion of the strip is determined by the relative decrease of the mean pair density $\delta n_{s} / n_{s}$. In the absence of perturbations, pairs would have the same velocity at a larger current $I_{1}=I\left(1-\delta n_{s} / n_{s}\right)$. Therefore, we suggest that the photon count rate at $I<I^{*}$ equals the dark count rate at the current $I_{1}=I\left(I_{C} / I^{*}\right)$.

Thin solid lines in Fig. 4 simulate the photon count rates beyond the cut-off. They were obtained by scaling down the current dependence of the dark count rate (dotted line in Fig. 4). The scaling factor was used as the only fitting parameter. Visually, the scaling factor appears as the normalized current corresponding to the cross-point of the fitting line and the level of the photon count rate at $I=I_{C}$ (straight horizontal line in Fig. (4). These values are marked with arrows and were used for the estimate of the QP multiplication efficiency, excluding the one for a wavelength of $0.8 \mu \mathrm{m}$. The method holds as long as the number of electron pairs destroyed by the photon in the $\xi$-slab is not very large or, equivalently, the number of remaining pairs compares to their equilibrium number. If the pair density changes noticeably, the current dependence of the fluctuation probability will change like it does with temperature. We believe that breaking of this condition did not allow us to satisfactorily fit the photon count rate for the most energetic quanta. An obvious spin-off of this approach is an understanding of the spectral dependence of the photon count rate beyond the cut-off. The spectral dependence should repeat the temperature dependence of the dark count rate at a fixed bias current. Available experimental data 2.13 qualitatively confirm this conclusion.

\section{ENERGY RESOLVING CAPABILITY}

The normal conducting domain created in the meander by the joint action of a photon and the bias current relaxes via thermal phonons, which pass the excess energy to the substrate. Since the relaxation rate is fixed, the life-time of the resistive state in the meander and, correspondingly, the duration of the voltage pulse increases with the full excess energy deposited in the film by the perturbation. Thus the duration of the voltage pulse should correlate with the energy of the photon, which initiated the formation of the resistive state. In this section we show that the resistive state indeed memorizes the photon energy.

A current-voltage curve of the $\mathrm{NbN}$ meander recorded in the voltage-bias regime at $2 \mathrm{~K}$ is shown in Fig. 7 After the critical current value is reached, the current drops signaling the formation of a normal domain. The point A corresponds to the smallest stable domain, which has a length of the order of the thermal healing length $L_{T}=\left(D \tau_{E}\right)^{1 / 2}$ where $\tau_{E}$ is the effective electron cooling time. When the voltage increases further, a plateau occurs at an almost constant current $I_{m}$ that maintains the domain in equilibrium. According to Ref $\underline{16}$, exactly at this current in a current biased meander the velocity of the domain walls would equal zero. The Stekly parameter, which is a measure of the self-heating by the bias current, can be evaluated as $\alpha \approx\left(I_{C} / I_{m}\right)^{2}=25$. Using the resistivity of our specimen and the dc resistance $\approx 1.2 \mathrm{k} \Omega$ corresponding to point $\mathrm{A}$, we estimate a thermal healing length $L_{T} \approx 60 \mathrm{~nm}$. With the diffusivity of $3.5 \cdot 10^{-5} \mathrm{~m} \mathrm{sec}^{-1}$ this length implies an effective electron cooling time $\tau_{E} \approx 100 \mathrm{ps}$. Using this electron cooling time, the measured critical current density $5.5 \cdot 10^{9} \mathrm{~A} \mathrm{~m}^{-2}$ and the electron specific heat $c_{E} \approx 2 \cdot 10^{2} \mathrm{~J} \mathrm{~m}^{-3} \mathrm{~K}^{-1}$, we independently estimated the value of the Stekly parameter as it follows from the dynamic theory ${ }^{16}$ of an 


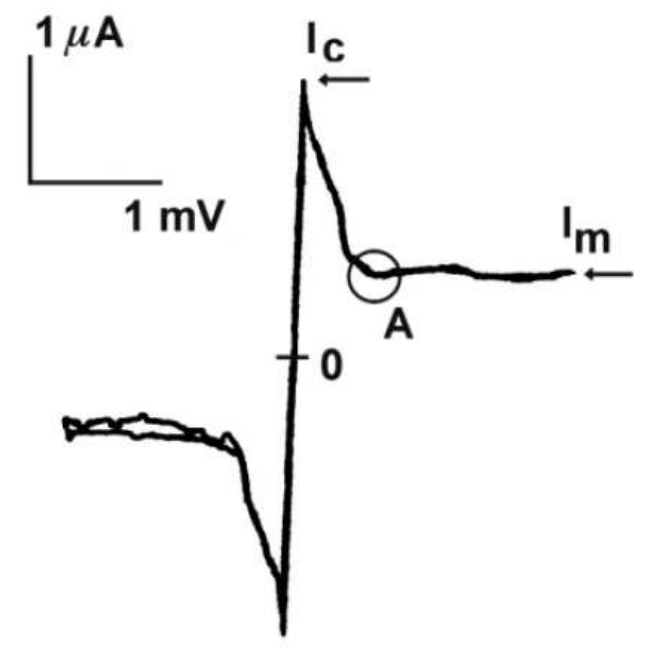

FIG. 7: Current-voltage characteristic of the $\mathrm{NbN}$ meander at $2 \mathrm{~K}$. The critical current $I_{C}$ and the current $I_{m}$ equilibrating the walls of the normal domain are both marked by arrows. The area A (marked by the circle) corresponds to the smallest stable normal domain of a size approximately equal to the thermal healing length.

electro-thermal domain $\alpha=\rho j_{C 2} \tau_{E} / c_{E}\left(T_{C}-T\right)$. We found $\alpha \approx 34$ in fair agreement with the value concluded from the $I-V$ curve. The steady-state parameters deliver a self-consistent description of the electro-thermal domain in our specimen ensuring reliability of the following consideration.

The Stekly parameter $\alpha \gg 1$ implies that even when $I \ll I_{C}$ self-heating strongly influences the dynamics of the normal domain. The critical energy that initiates the thermal roll-off or quenching in a current-biased meander can be presented ${ }^{16}$ as

$$
\begin{gathered}
Q_{0}=L_{T} w d c_{E}\left(T_{C}-T\right) \alpha i^{2} \ln \left(\frac{\alpha i^{2}}{\alpha i^{2}-2 \theta}\right), \\
\text { with } \theta=\frac{T_{C}(I)-T}{T_{C}-T} \text { and } i=\frac{I}{I_{C}},
\end{gathered}
$$

where $T_{C}(I)$ denotes the temperature, for which the critical current $I_{C}(T)$ equalizes the bias current $I$. Assuming the standard temperature dependence of the critical current, we find for the $\mathrm{NbN}$ meander at $I / I_{C}=0.8 \mathrm{a}$ critical energy $Q_{0}=0.51 \mathrm{eV}$ that is $\approx 3$ times smaller than the energy of a $0.8-\mu \mathrm{m}$ photon. Depending on the time constant of the bias and on the strength of the negative feedback, the quenching may partly occur also in the voltage-bias regime. To fully avoid quenching the time constant of the bias should be noticeably smaller than the electron cooling time. The rates of photon and dark counts at different biases are shown in Fig. (4) The highest count rate of $\approx 5 \cdot 10^{7} \mathrm{sec}^{-1}$ was measured at $I \approx 0.98 I_{C}$. Such count rate corresponds to a recovery time of $\approx 20$ ns that coincides with the full duration of the signal transient seen in Fig. 1. We believe that the recovery time more than 10 times larger than the bias

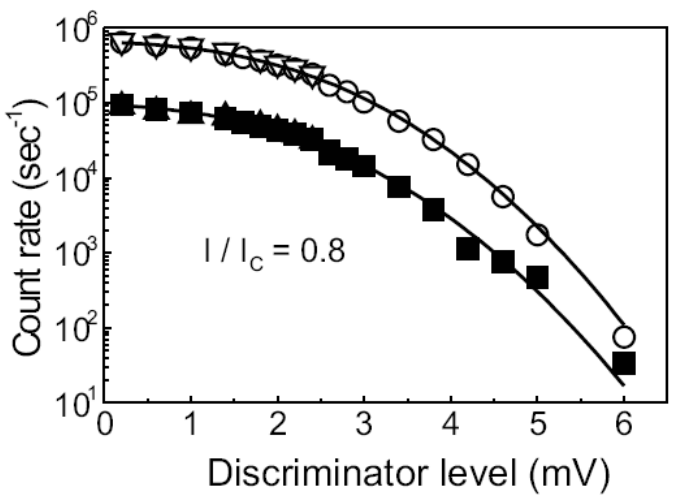

FIG. 8: The rate of dark (closed symbols) and photon (open symbols) counts at different discriminator levels. Solid lines are best fits assuming normal statistical distributions of the pulse amplitudes.

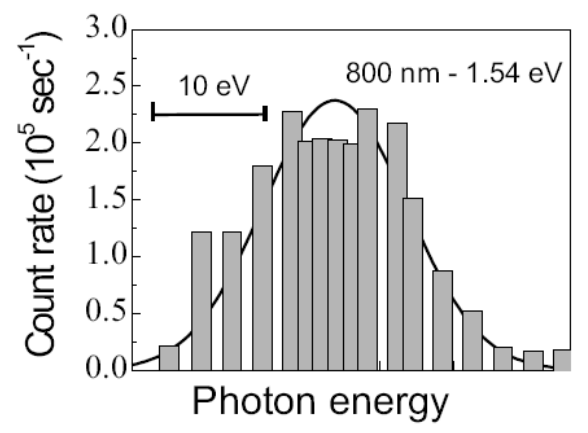

FIG. 9: Spectrum of $0.8-\mu \mathrm{m}$ photons recorded with the $\mathrm{NbN}$ meander at $2 \mathrm{~K}$.

time constant $\tau_{B} \approx 0.5$ ns was most likely determined by the lower edge of the amplifier band-pass.

At large bias currents very close to $I_{C}$ the noise background may be so high that dark counts outnumber photon counts. Accordingly, uncertainties in the photon count rate increase. Therefore, we analyzed the statistical distribution of the voltage transients at a smaller bias $I=0.8 I_{C}$ when the rate of dark counts was at least an order of magnitude less than the rate of photon counts. Fig. 8 shows the dark count rate and the photon count rate for $0.8-\mu \mathrm{m}$ photons measured at different discriminator levels of the pulse counter. When the discriminator level is set low all events are counted. Increase of the discriminator level leaves more and more events uncounted until at a high enough level none is counted anymore. Assuming a normal distribution of the signal amplitude, we fit the integral of the distribution to the experimental data. Best-fit curves are shown in Fig. 8 Two fitting parameters were used: the mean value and the dispersion of the normal distribution. We have found a best dispersion value $1.65 \pm 0.03 \mathrm{mV}$ for both dark and photon counts and mean values $1.7 \mathrm{mV}$ 
and $1.9 \mathrm{mV}$ for dark and photon counts, respectively. In terms of the strength and statistical distribution, dark counts are identical to photon counts when photons have the critical energy $Q_{0}$. The wavelength of a photon having this critical energy is $2.4 \mu \mathrm{m}$ perfectly matching the longest wavelength we detected with this meander. Relating the mean values of the signal amplitudes to the energies of $0.8-\mu \mathrm{m}$ and $2.4-\mu \mathrm{m}$ photons we found a scaling factor of $0.14 \mathrm{mV} / \mathrm{eV}$. Fig. 9 presents the spectral histogram for $0.8-\mu \mathrm{m}$ photons retrieved with this scaling factor from the experimental data. The best-fit envelope shown by the solid line represent a normal distribution with a dispersion of $6.5 \mathrm{eV}$. Although the effect has an energy resolving power, it is far too low for practical purposes. Factors limiting the energy resolution are discussed below.

We should first estimate the energy dissipated in the meander by the bias current. After a photon has released an energy larger than $Q_{0}$, the normal domain starts to grow. The propagation velocity of the domain walls, i.e. the superconductor-normal interfaces, determines the growth rate of the normal domain. The velocity decreases as the actual bias current drops following the domain growth. In our experiment the life-time of the domain roughly coincides with the time constant $\tau_{B}$ of the bias circuit. The size of the domain, and consequently the energy dissipated by the current, can be determined integrating the Joule power with the particular current dependence of the interface velocity. Considering the simple step-edge heat-dissipation model, it can be shown ${ }^{16}$ that the interface velocity $\mathrm{v}$ increases linearly with the current:

$$
v=\left(\alpha \frac{D}{\tau_{E}}\right)^{1 / 2}\left(i-i_{m}\right) \theta^{-1 / 2},
$$

where $i_{m}=I_{m} / I_{C}$. With this dependence the expression for the Joule energy dissipated within the time $\tau_{B}$ takes the form

$$
E_{B}=\frac{1}{5} I^{2} \rho\left(\frac{D}{\tau_{E}}\right)^{1 / 2} \frac{\tau_{B}^{2}}{w d} .
$$

Using $\tau_{B} \approx 0.5 \mathrm{~ns}$ we found a current contribution of $\approx 11 \mathrm{eV}$ that is almost an order of magnitude larger than the energy of a $0.8-\mu \mathrm{m}$ wavelength photon. Given the linear dependence of the response duration on the total energy released in the meander, the ratio of the mean amplitudes of photon and dark counts (see Fig. 8) correlates well with the energy contributed by the bias. Denoting by $\epsilon_{1}$ and $\epsilon_{2}$ the energies of $0.8-\mu \mathrm{m}$ and $2.4-\mu \mathrm{m}$ photon, respectively, we find $\left(E_{B}+\epsilon_{1}\right) /\left(E_{B}+\epsilon_{2}\right) \approx 1.09$ in agreement with the amplitude ratio 1.1 concluded from the experiment.

The dispersion of the life-time of the normal domain may originate from structural defects of the meander mediated by the domain length. In $\mathrm{NbN}$ films defects are most likely boundaries between grains, which have a typical size of ten nanometers ${ }^{17}$. Intuitively, the dispersion has a maximum value when the domain length is of the order of the grain size. Contribution of the film structure to the measured dispersion seems plausible because the normal domain in our meanders covers only a few grains. The cooled amplifier also contributed to the measured dispersion since its equivalent voltage noise was only slightly less than the amplitude of the voltage pulse delivered by the meander. With a SQUID amplifier that typically has a subkelvin noise temperature, the energy resolution should drastically improve. Finally, using a superconductor with a smaller energy gap one should be able to increase the conversion ratio of the photon energy to the domain length. Given the same dispersion this will also lead to a better energy resolution. An estimate shows that the cumulative effect of all factors mentioned should suffice for an energy resolution comparable to the resolution of conventional superconductor photon detectors.

In summary, we observed the current dependent spectral cut-off in the appearance of the resistive state after absorption of a single near infrared photon in nanostructured $\mathrm{NbN}$ superconducting films. We generalized the normal-spot model explicitly taking into account the diffusion profile of nonequilibrium quasiparticles. With this approach, we found a super-linear photon energy dependence of the quantum yield in $\mathrm{NbN}$ that was attributed to a splitting of the thermalization process into the phonon and the electron cascades around the Debye energy. The resistive state was shown to memorize the energy of the photon, which had initiated it. Ultimate energy resolving capability of this effect should be comparable with the energy resolution of conventional detectors.
* Physics Institute of the University of Zurich, Winterthurerstr. 190, 5087 Zurich, Switzerland

1 A. Semenov, G. Gol'tsman, A. Korneev, Physica C 351, (2001) 349-356.

2 G.N. Gol tsman, O. Okunev, G. Chulkova, A. Lipatov, A. Semenov, K. Smirnov, B. Voronov, C. Williams, and R. Sobolevski, Applied Physics Letters 79, (2001) 705-707.

3 A.Verevkin, J. Zhang, R. Sobolewski, A.Lipatov, O. Okunev, G. Chulkova, A. Korneev, K. Smirnov, G.
Gol'tsman, and A. Semenov, Applied Physics Letters 80, (2002) 4687-4689.

4 J. Zhang, W. Slysz, A. Pearlman, A. Verevkin, R. Sobolewski, O. Okunev, G. Chulkova, and G.N. Gol'tsman, Phys. Rev. B 67, (2003)132508.

5 A.G. Kozorezov, A.F. Volkov, J.K. Wigmore, A. Peacock, A. Poelaert, and R. den Hartog, Phys. Rev. B 61, (2000) 11807-11819.

6 A. Semenov, A. Engel, K. Il'in, G. Gol'tsman, M. Siegel, 
and H-W. Hübers, The European Physical Journal - Applied Physics 21, (2003) 171-178.

7 L.N. Cooper, Phys. Rev. Lett. 6, (1961) 689-690.

8 Direct measurements of the magnetic penetration length indicated normal conducting $\approx 0.4 \mathrm{~nm}$ thick layers at the film interfaces with the substrate and air and also $\approx 4$ $\mathrm{nm}$ wide normal conducting areas along the stripe edges. Together with the observed $15 \%$ non-homogeneity of the stripe width this reduces the electrical cross-section of our meanders to 0.58 of the geometrical cross-section.

9 M.R. Beasley, J.E. Mooij, and T.P. Orlando, Phys. Rev. Lett. 42, (1979) 1165-1168.

10 M. Tinkham, Introduction to Superconductivity (McGrawHiii, Inc., New York 1996) 288-293.

11 C.N. Lau, N. Markovic, M. Bockrath, A. Bezryadin, and
M. Tinkham, Phys. Rev. Lett. 87, (2001) 217003-1-4.

12 A. Garzarella and C.J. Martoff, J. Appl. Phys. 79, (1996) 2426-2434.

13 A. Engel, A. Semenov, H.-W. Hübers, K. Il'in, and M. Siegel, J. Mod. Optics 51, (2004)1459.

14 R.F. Voss, C.M. Knoedler, and P.M. Horn, Phys. Rev. Lett. 45, (1980) 1523-1526.

15 A. Anthore, H. Pothier, and D. Esteve, Phys. Rev. Lett. 90, (2003) 127001.

16 A.V. Gurevich and R.G. Mints, Reviews of Modern Physics, 59, (1987) 941-999.

17 A. Semenov, H. Richter, K. Smirnov, B. Voronov, and H.W. Hübers, Supercond. Sci. Technol. 17, (2004) 1-4. 